\title{
Collaborative stepped care $v$. care as usual for common mental disorders: 8-month, cluster randomised controlled trial ${ }^{\dagger}$
}

Desiree B. Oosterbaan, Marc J. P. M. Verbraak, Berend Terluin, Adriaan W. Hoogendoorn, Wouter J. Peyrot, Anna Muntingh and Anton J. L. M. van Balkom

\section{Background}

Thus far collaborative stepped care (CSC) studies have not incorporated self-help as a first step.

\section{Aims \\ To evaluate the effectiveness of CSC in the treatment of common mental disorders.}

\section{Method}

An 8-month cluster randomised controlled trial comparing CSC to care as usual (CAU) (Dutch Trial Register identifier NTR1224). The CSC consisted of a stepped care approach guided by a psychiatric nurse in primary care with the addition of antidepressants dependent on the severity of the disorder, followed by cognitive-behavioural therapy in mental healthcare.

\section{Results}

Twenty general practitioners (GPS) and 8 psychiatric nurses were randomised to provide CSC or CAU. The GPS recruited 163 patients of whom 85\% completed the post-test measurements. At 4-month mid-test CSC was superior to CAU: $74.7 \%(n=68)$ v. $50.8 \%(n=31)$ responders $(P=0.003)$. At 8-month post-test and 12-month follow-up no significant differences were found as the patients in the CAU group improved as well.

\section{Conclusions}

Treatment within a CSC model resulted in an earlier treatment response compared with CAU.

\section{Declaration of interest}

None.
Although most patients with common mental disorders receive treatment in primary care, ${ }^{1}$ there is room for improvement in the standard of mental healthcare in this setting. ${ }^{2}$ Multifaceted interventions that target patients, providers and system changes have been proposed to improve the quality of treatment for these conditions in primary care. ${ }^{3,4}$ The collaborative care model incorporates multiple interventions and has been extensively studied in the field of depression treatment ${ }^{5}$ and to a lesser extent for anxiety, ${ }^{5,6}$ yielding positive results. Clinical guidelines are increasingly focusing on the efficient provision of mental healthcare, making stepped care an attractive treatment model, starting with a minimal intervention such as (guided) self-help in primary care and stepping up to specialised mental healthcare only when necessary. ${ }^{7,8}$ Thus far collaborative care studies have not incorporated stepped care with (guided) self-help as a first step. Furthermore, most research on collaborative care has targeted patients with depressive disorders, but anxiety disorders and stress-related disorders are also frequently seen in primary care and might also be treated effectively with collaborative care. ${ }^{5,9}$ Therefore, a study examining the effectiveness of a collaborative stepped care (CSC) approach for common mental disorders is needed. Because CSC intervenes at the level of the primary care practice, we used a pragmatic cluster randomised controlled trial (RCT) to evaluate response to treatment and remission in patients with depressive, anxiety or stress-related disorders receiving CSC or care as usual (CAU).

\section{Method}

\section{Design}

This Dutch 8-month practice-based study with a 4-month follow-up phase was designed to evaluate the clinical outcomes of CSC as compared with CAU in the treatment of common

\footnotetext{
†See editorial, pp. 86-87, this issue.
}

mental disorders. The CSC treatment algorithm included both psychological and pharmacological stepwise interventions for the treatment of patients with anxiety, depressive and stress-related disorders in both primary care and specialised mental healthcare. Measurements took place mid-test at 4 months, post-test at 8 months and at 12-month follow-up. The study was approved by the Medical Ethics Committee of the Twenteborg Hospital in Almelo (Dutch Trial Register identifier NTR1224, www. trialregister.nl).

\section{Eligibility criteria for clusters and participants}

\section{Clusters}

All 30 general practitioners (GPs) established in the region where the specialised mental healthcare centre is located were contacted for participation. Inclusion criteria were: (a) the GPs were working with a psychiatric nurse; and (b) giving informed consent. After a complete explanation of the study procedures, informed consent was obtained and the GPs were randomised to one of the two conditions: CSC or CAU. In addition, two teams were formed within the specialised mental health centre, each consisting of four psychiatric nurses, two psychologists and one psychiatrist. The teams were randomised to one of the two conditions: CSC or CAU. The randomisation codes were developed by an independent statistician. The details of the series were unknown to any of the researchers.

\section{Patients}

Inclusion criteria. Included were out-patients aged 18 years or older with (a) common mental disorders diagnosed by the GP and (b) who gave written informed consent to participate. Included were patients with the following DSM-IV ${ }^{10}$ disorders: panic disorder, agoraphobia, social phobia, specific phobia, generalised anxiety disorder, unipolar major and minor depressive 
disorder, dysthymia or one of the stress-related adjustment disorders.

Exclusion criteria. Patients were excluded when they met the DSM-IV ${ }^{10}$ criteria for dependence on alcohol or drugs, dementia, psychotic disorder or bipolar disorder. Patients with posttraumatic stress disorder and obsessive-compulsive disorder were included only when these disorders were comorbid with one of the disorders in the inclusion criteria. Furthermore, patients were excluded if they were already undergoing treatment with psychotropic drugs (except for benzodiazepines), cognitive-behavioural therapy (CBT) or interpersonal therapy for the present episode of the disorder. In addition, patients were excluded if they were not sufficiently proficient in the Dutch language to fill in the measurement scales.

\section{Procedure}

Patients who were selected for the study by the GP were visited at the office of the GP by a trained research psychologist. The research psychologist administered the Mini International Neuropsychiatric Interview (MINI) ${ }^{11}$ which is a structured psychiatric interview that diagnoses DSM-IV disorders. ${ }^{10}$ The purpose of this diagnostic interview was (a) to determine the primary DSM-IV diagnosis in case of psychiatric comorbidity and (b) to formally assess the exclusion criteria. In patients with multiple disorders the primary diagnosis was defined as the psychiatric disorder from which the patient suffered the most. This primary diagnosis determined the allocation of the patients to one of the three different treatment algorithms (one for anxiety disorders, one for depressive disorders and one for stress-related disorders; see Fig. 1).

\section{Interventions}

\section{Care as usual}

The patients treated by the GPs assigned to CAU could obtain any service normally available in The Netherlands. Thus, CAU could encompass no treatment, the prescription of antidepressants,

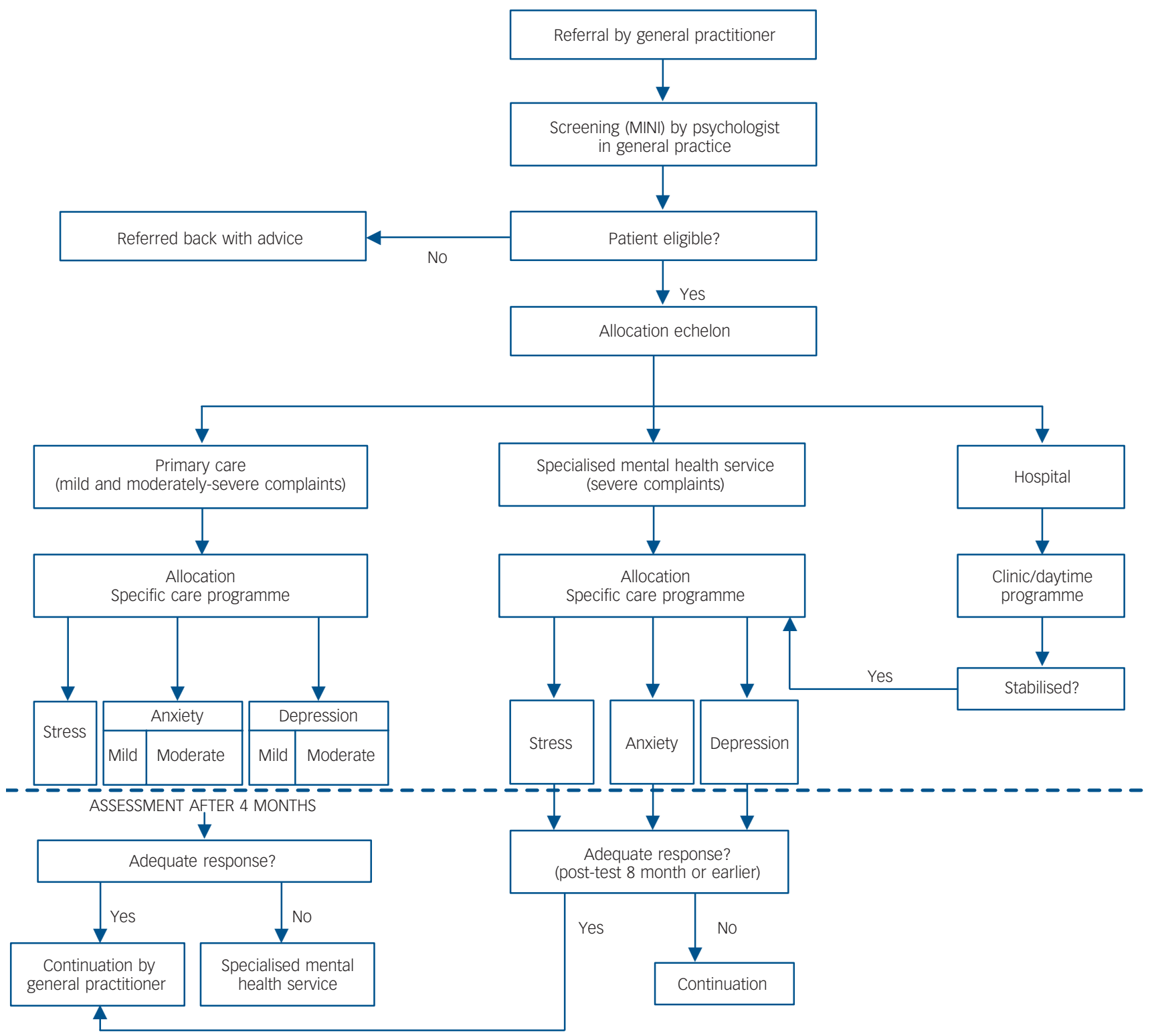

Fig. 1 Outline of the collaborative stepped care programme.

MINI, Mini International Neuropsychiatric Interview. 
referral to a psychiatric nurse at the GP's office, referral to the specialised mental health centre or to another professional. When the patients were referred to the specialised mental health centre, treatment could consist of any type of pharmacotherapy, psychotherapy or a combination of the two. In The Netherlands, national guidelines for the treatment of depression and anxiety disorders are available for GPs and psychiatrists. However, adherence with these guidelines is not optimal. ${ }^{12}$

\section{Collaborative stepped care}

The patients treated by the GPs assigned to CSC were offered treatment according to three different treatment algorithms: one for anxiety disorders, one for depressive disorders and one for stress-related disorders. The algorithms were described in a manual, including interventions and treatment decisions (Fig. 1).

Treatment programmes. All patients with stress-related disorders started with the first step in primary care. For the anxiety and depression treatment programmes, the level of severity of the disorder was determined by the number of symptoms, the severity of symptoms and the degree of functional impairment as assessed by the psychologist who conducted the MINI interviews. Patients with mild or moderately severe anxiety or depressive disorders started treatment with a first-step treatment in primary care. Patients with a severe disorder were directly referred to the out-patient department of the specialised mental health service for the second-step treatment and were offered CBT and antidepressants. In cases when patients had depression with psychotic features, were actively suicidal or when the family of the patient was overly strained due to the psychiatric disorder, the patients were referred to a (day care) clinic where they received intensive psychiatric treatment.

First- and second-step interventions. The first-step intervention was provided in primary care. Patients were offered a 3.5-month self-help course, with guidance from the psychiatric nurse at the GP's office or at home, in five 45-minute sessions. The self-help course for depression was a widely used course based on the method of Lewinsohn and colleagues. ${ }^{13}$ The self-help course 'Stresspac' was used for anxiety and stress-related disorders. ${ }^{14}$ Both courses consist of workbooks with psychoeducation and cognitive and behavioural exercises and have been shown to be efficacious in previous studies. ${ }^{14,15}$

In addition to guided self-help, patients with a moderately severe anxiety or depressive disorder were offered antidepressant medication according to disorder-specific algorithms, initially with a selective serotonin reuptake inhibitor (SSRI). In case of non-response or low tolerability, in depressive disorder the firststep SSRI was switched to mirtazapine, venlafaxine or a tricyclic antidepressant. In panic disorder and social phobia the first-step SSRI was switched to another SSRI, whereas in generalised anxiety disorder this second step could also mean a switch to venlafaxine.

Treatment offered in step two consisted of CBT in combination with antidepressants provided in the out-patient specialised mental health service.

Monitoring. Remission was evaluated after 4 months (Fig. 1) by the psychiatric nurse, using the Clinical Global Impression Severity scale (CGI-S). ${ }^{16}$ Patient with scores $\geqslant 3$ on the CGI-S (i.e. mild severity or worse) proceeded to the second-step treatment: referral to a specialised mental health service. The evaluation by the psychiatric nurse in CSC was used only to evaluate treatment progression and to determine whether or not to 'step up' treatment. In contrast, the outcome of CSC and
CAU was evaluated at 4,8 and 12 months by an independent, trained rater (see Measurements).

Training. To enhance the adherence of the professionals in the CSC group to the algorithms, multiple tools were provided at the cluster level. The GPs received one educational session by a psychiatrist to clarify the medication algorithm and to advise on enhancing medication adherence. During the study there was an opportunity for direct, case-based consultation with the psychiatrist who was randomised to CSC. This consultation occurred about once per 2 months. Psychiatric nurses participated in a 2-day training session in basic CBT strategies. They had a detailed manual, structured session-by-session, at their disposal to guide the self-help course. In addition, group supervision for feedback and adherence to the manual took place every 2 weeks with an experienced behavioural therapist. The psychologists who provided the second-step treatment in the specialised mental health service used CBT session-by-session protocols commonly used in The Netherlands, which have been proven efficacious in clinical studies. The psychiatrist used the same pharmacotherapy algorithm used by the GP.

\section{Measurements}

All outcome measures pertained to the level of the patients. Patients were assessed at baseline, at a 4-month mid-test, an 8 -month post-test and at a 12-month follow-up with self-ratings or with assessor ratings by independent, trained raters, who were masked to the condition to which the GPs had been allocated (interrater reliability was not assessed). When patients stopped treatment prematurely, intensive effort was made to complete all measurements. In this study we distinguished two types of individuals who dropped out: (a) those who dropped out from treatment; and (b) those for whom measurements could not be completed, i.e. who dropped out of providing data.

\section{Primary outcome measures}

Primary outcome measures were the percentage of patients responding to and remitting after treatment. Response was defined as a score of 1 ('very much improved') or 2 ('much improved') on the assessor-rated Clinical Global Impression of Improvement Scale (CGI-I) as measured from baseline. ${ }^{16}$ Remission was defined as a score of 1 ('normal, not at all ill') or 2 ('borderline mentally ill') on the assessor-rated CGI-S. ${ }^{16}$ Both CGI-I and CGI-S were chosen because they are generic outcome measures that assess severity and change in psychopathology irrespective of its nature. However, disorder-specific, dimensional secondary outcome measures were used in addition.

\section{Secondary outcome measures}

Secondary outcome measures included disorder-specific dimensional scales, used to evaluate the severity of symptoms, patient functioning and satisfaction with treatment. The assessor-rated Hamilton Rating Scale for Anxiety (HRSA) was used to measure the presence and severity of anxiety symptoms. ${ }^{17}$ Depressive symptoms were measured with the self-rated Center for Epidemiologic Studies Depression Scale (CES-D). ${ }^{18}$ The selfrated Fear Questionnaire $(\mathrm{FQ})^{19}$ was used to assess phobic avoidance behaviour, the self-rated Symptom Checklist-90Revised (SCL-90-R) was used to measure the severity of general psychoneurotic symptoms, ${ }^{20}$ and the self-rated Short-Form 36 Health Survey (SF-36) ${ }^{21}$ mental scale was used to measure quality of life.

The patient's perspective was measured by having patients rate patient versions of the CGI-I and CGI-S at each point. 


\section{Power considerations and sample size}

The aim of the RCT was to detect a clinically relevant $25 \%$ difference in response between CSC and CAU at the 8-month follow-up. We assumed we would find the following response percentages: CSC $75 \%$ v. CAU 50\%, corresponding to Cohen's $h=0.52$. With a two-sided alpha of 0.05 , and a beta of 0.20 , a sample of 58 patients per condition was required. ${ }^{22}$ The estimated difference of $25 \%$ was not based on previous data on collaborative care effectiveness. At the time the study was designed, no data on CSC were available in The Netherlands. We based this estimation on a clinically significant difference that we thought would be relevant for clinical practice.

We estimated a drop-out rate of $25 \%$ from the clusters of eight participants (on average) per GP and an intraclass correlation of $5 \%$. The intraclass correlation coefficient of $5 \%$ was chosen based on previous experiences with primary care studies. It appeared to be consistent with an analysis of cluster-based studies in primary care by Adams and colleagues. ${ }^{23}$ Thus, a total of 96 patients per treatment arm were required. During the study, recruitment in the CAU condition was slower than expected. Also, the drop-out rate appeared to be lower than expected. The final number of patients included, 94 in the CSC condition and 64 in the CAU condition, revealed a power of 0.80 to detect an increase in response rate from 50 to $75 \%$ with a two-sided significance level of 0.05 .

\section{Statistical methods}

We used statistical procedures appropriate to the cluster and longitudinal design. For the primary outcome measures multilevel logistic regression models were used to correctly estimate the differences in response and remission between groups (CSC $v$. $\mathrm{CAU})$ taking the clustering of patients within general practices (as a single level) into account by modelling the clustering as random effects. Statistical significance was evaluated using the $z$-statistic. Analysis of the primary outcome measures was conducted on the intention-to-treat sample using last observation carried forward, thereby assuming that patients who dropped out did not respond or remit.

For the secondary measures, a mixed-effects model approach was applied on the intention-to-treat sample. The model included measurement (baseline, 4-month mid-test, 8-month post-test and 12-month follow-up), treatment and the measurement treatment interaction as fixed effects, and GPs (first level) and patients (second level) as random effects. All tests of treatment effects were conducted at a two-sided alpha level of 0.05. Data were analysed using SPSS 13.2 and Stata 11.0 for Windows.

\section{Results}

\section{Characteristics and attrition for clusters and participants}

\section{Clusters}

Of 30 GPs who were contacted for participation, 8 refused. Reasons for refusal included an unwillingness to risk allocation to CAU $(n=5)$, unwillingness to invest the necessary time $(n=2)$ and lack of room for a psychiatric nurse $(n=1)$. Twentytwo GPs, eight psychiatric nurses, four psychologists and two psychiatrists working at the community mental health centre were randomly assigned to either CSC or CAU. Another two GPs refused to participate after randomisation to CAU, resulting in ten GPs randomised to each condition (Fig. 2). Clusters ranged from 1 to 25 included patients (mean number of patients per cluster 7.9 (s.d. $=7.5)$ ).

\section{Patients}

The GPs recruited 163 patients between April 2003 and July 2005, however 5 individuals did not return the baseline questionnaires and were therefore excluded from subsequent analysis. The GPs in the CSC condition had 94 patients $v$. 64 patients in the CAU condition. The most common primary diagnosis was a depressive disorder $(n=73)$, followed by an anxiety disorder $(n=57)$ and a stress-related disorder $(n=28)$.

\section{Drop-out rates}

Demographic characteristics and clinical status variables of the patients at baseline are shown in Tables 1 and 2. Data on primary outcome measures were obtained from 152/158 (96\%) of the patients at the 4-month mid-test, 139/158 (88\%) at the 8-month post-test and $127 / 158(80 \%)$ at the 12-month follow-up. Patients with complete data did not differ significantly from those who dropped out of providing data with respect to demographic and clinical variables.

Treatment drop-out from the CSC algorithm as well as CAU was defined as ceasing contact with the care provider prematurely and without consultation for 3 months or more before the 8-month post-test, while psychiatric complaints were not in remission. The treatment drop-out percentage was $14.9 \%(n=14)$ in the CSC group v. $7.8 \%(n=5)$ in the CAU condition $(z=1.13, P=0.257)$. In the CSC group, significantly more patients dropped out from the anxiety treatment programme compared with the depression treatment programme $(31.0 \%(n=9)$ v. $8.5 \%(n=4), z=2.29$, $P=0.022)$. Patients who left treatment prematurely had a significantly higher number of mean diagnoses (mean 3.1 v. 2.1, $z=3.21, P=0.001$ ), a significantly longer duration of their main diagnosis (mean 6.7 v. 2.8 years, $z=2.07, P=0.041$ ) and were significantly younger in age (mean 37.9 v. 32.0 years, $z=2.07$, $P=0.039$ ).

\section{Collaborative stepped care treatment}

According to the CSC protocol, 88 (94\%) of the 94 patients in the CSC group were allocated to guided self-help, the first-step treatment. Six patients $(6 \%)$ in the CSC group had a severe disorder and were thus directly referred to second-step treatment (specialised mental healthcare). Of the 88 patients allocated to the first-step treatment, 9 patients (10\%) never started guided self-help. Of the remaining 79 patients who started guided self-help, 29 patients $(37 \%)$ had a mild to moderately severe disorder and were offered guided self-help; 50 patients $(63 \%)$ had a moderately severe disorder and were offered guided self-help plus antidepressant medication. However, not all patients accepted medication. Of the 79 patients who started guided self-help, 44 (56\%) achieved full remission. The remaining 35 patients (44\%) did not respond to step one. Of these, 20 (25\% of the patients who started guided self-help) started step two and were offered CBT and pharmacotherapy in the specialised mental healthcare centre, and 15 (19\%) declined further treatment. Another seven patients who did achieve remission required further specialised mental healthcare treatment because of additional mental health issues. Overall, 27 (34\%) of the 79 patients who started the first-step treatment continued their treatment in the specialised mental healthcare centre.

\section{Care as usual}

Of the 64 patients in the care as usual group, 5 terminated treatment prematurely (defined as having no contact with a care provider for 3 months or more in the first 8 months of treatment, while psychiatric symptoms were not yet in remission). In total 


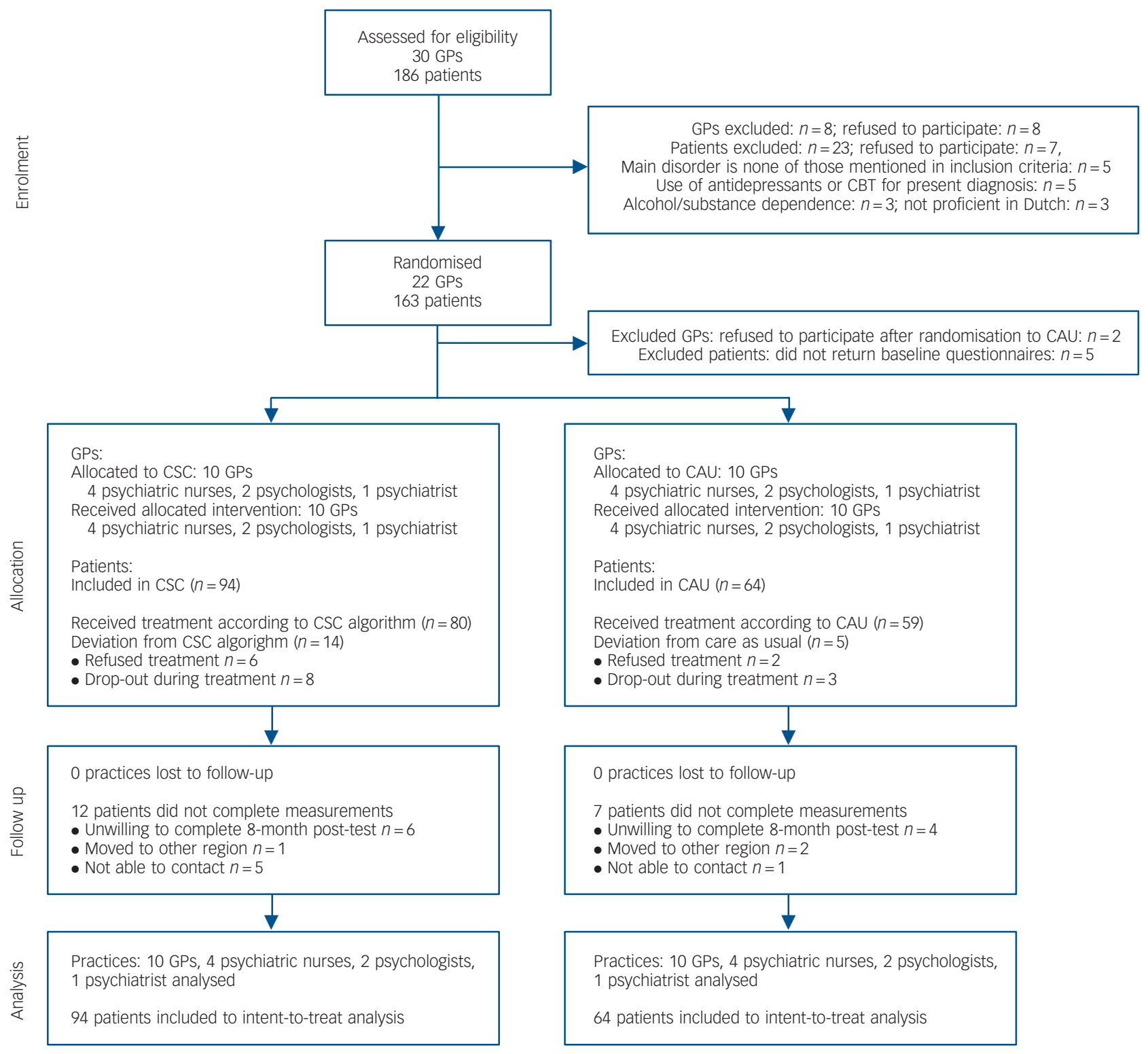

Fig. 2 Diagram showing the flow of clusters and patients through the trial.

$\mathrm{CAU}$, care as usual; CBT, cognitive-behavioural therapy; CSC, collaborative stepped care; GP, general practitioner.

$37 \%(n=23)$ of the patients were prescribed antidepressants and $44 \%(n=28)$ of the patients were referred to specialised mental healthcare.

\section{Primary outcome measures}

Collaborative stepped care led to higher response and remission rates compared with CAU at 4, 8 and 12 months (see online Figs DS1 and DS2). At the 4-month mid-test, significantly more patients in the CSC group compared with the CAU group responded $(74.7 \%(n=68)$ v. $50.8 \%(n=31), z=2.99, P=0.003)$ and remitted $(57.8 \% \quad(n=52) \quad$ v. $31.7 \% \quad(n=19), \quad z=3.09$, $P=0.002$ ). At the 8 -month post-test the CAU group improved and differences were no longer significant (response $80.2 \%$ ( $n=73)$ v. $67.2 \%(n=41), z=1.80, P=0.072$; remission $58.9 \%$ $(n=53) \quad$ v. $51.7 \% \quad(n=31), \quad z=0.87, P=0.383)$. At 12 -month follow-up, differences diminished further (response $83.5 \%$ $(n=76)$ v. $77.0 \%(n=47), z=0.99, P=0.322)$; remission $73.3 \%$ $(n=66) \quad$ v. $61.7 \% \quad(n=37), \quad z=1.50, P=0.133)$, because the patients in the CAU improved further.
Results for the anxiety and depression programmes are presented in Table 3. The number of patients allocated to the stress treatment programme was too small to permit a separate analysis. In both the anxiety and depression treatment programmes the response and remission rates of the CSC group were higher than those of the CAU group at the measurement points. However, significant differences were found only at the 4-month mid-test.

\section{Secondary outcome measures}

Compared with patients in the CAU group, CSC patients had a significantly larger reduction in anxiety symptoms (HRSA, FQ) and depressive symptoms (CES-D) after the 4-month mid-test (Table 2). No significant differences were found on the other secondary outcome measures when considering the whole group of patients. In the anxiety treatment programme scores on the HRSA were significantly more reduced in the CSC compared with the CAU group (online Fig. DS3). However, for the depression treatment programme we did not find any 


\begin{tabular}{|c|c|c|c|}
\hline Characteristics & $\begin{array}{l}\text { Collaborative stepped care group } \\
\qquad(n=94)\end{array}$ & $\begin{array}{l}\text { Care as usual group } \\
\qquad(n=64)\end{array}$ & $P$ \\
\hline \multicolumn{4}{|l|}{ Demographic } \\
\hline Age, years: mean (s.d.) & $37.0(11.8)$ & $39.4(12.4)$ & 0.23 \\
\hline Female, $n(\%)$ & $59(62.8)$ & $39(60.9)$ & 0.82 \\
\hline Higher education (college or university), $n$ (\%) & $19(20.2)$ & $15(23.4)$ & 0.65 \\
\hline Employed, $n(\%)$ & $73(77.7)$ & $43(67.2)$ & 0.13 \\
\hline Foreign, $n(\%)$ & $5(5.3)$ & $6(9.4)$ & 0.33 \\
\hline \multicolumn{4}{|l|}{ Clinical } \\
\hline Number of diagnosis, mean (s.d.) & $2.3(1.2)$ & $2.1(1.1)$ & 0.29 \\
\hline Duration, year, mean (s.d.) & $3.4(6.3)$ & $3.2(6.1)$ & 0.83 \\
\hline Psychiatric history, $n(\%)$ & $34(36.2)$ & $21(32.8)$ & 0.66 \\
\hline Severely ill, $n$ (\%) & $25(26.6)$ & $20(31.3)$ & 0.53 \\
\hline \multicolumn{4}{|l|}{ Main diagnosis, $n(\%)$} \\
\hline Any depressive disorder & $47(50.0)$ & $26(40.6)$ & 0.25 \\
\hline Any anxiety disorder & $29(30.9)$ & $28(43.8)$ & 0.10 \\
\hline Any stress-related disorder & $18(19.1)$ & $10(15.6)$ & 0.57 \\
\hline
\end{tabular}

\begin{tabular}{|c|c|c|c|c|}
\hline & \multicolumn{4}{|c|}{ Mean (s.d.) } \\
\hline & Pre-test & Mid-test (4 months) & Post-test (8 months) & Follow-up (12 months) \\
\hline \multicolumn{5}{|l|}{ Symptom Checklist-90-Revised } \\
\hline Collaborative stepped care & $190.27(46.96)$ & $140.07(41.76)$ & $133.62(40.86)$ & $128.27(41.01)$ \\
\hline Care as usual & $192.96(40.58)$ & $151.75(41.31)$ & $140.07(35.50)$ & $132.92(35.42)$ \\
\hline \multicolumn{5}{|l|}{ Hamilton Rating Scale for Anxiety } \\
\hline Collaborative stepped care & $20.44(8.13)$ & $9.71(7.57)^{\mathrm{a}}$ & $8.72(7.29)$ & $7.98(7.06)$ \\
\hline Care as usual & $20.92(6.96)$ & $13.59(7.49)$ & $10.88(6.25)$ & $8.90(6.04)$ \\
\hline \multicolumn{5}{|c|}{ Center of Epidemiologic Studies Depression Scale } \\
\hline Collaborative stepped care & $27.64(10.89)$ & $13.75(9.97)^{\mathrm{a}}$ & $12.82(9.91)$ & $11.22(9.81)$ \\
\hline Care as usual & $26.67(9.32)$ & $17.57(9.94)$ & $14.46(8.54)$ & $12.47(8.39)$ \\
\hline \multicolumn{5}{|l|}{ Fear Questionnaire } \\
\hline Collaborative stepped care & $24.29(16.88)$ & $16.96(14.12)^{\mathrm{a}}$ & $16.79(13.91)$ & $12.62(13.86)^{b}$ \\
\hline Care as usual & $22.45(14.60)$ & $21.90(14.03)$ & $18.61(12.02)$ & $16.04(11.90)$ \\
\hline \multicolumn{5}{|l|}{ Short-Form 36 Health Survey } \\
\hline Collaborative stepped care & $40.48(20.68)$ & $63.65(18.64)$ & $64.95(18.03)$ & $69.99(17.73)$ \\
\hline Care as usual & $40.43(17.86)$ & $60.01(18.42)$ & $70.10(15.62)$ & $68.89(15.34)$ \\
\hline
\end{tabular}

significant differences between the treatment groups on the CES-D (online Fig. DS4).

\section{Patient perspective}

At both the 4-month mid-test and 8-month post-test significantly more patients in the CSC group rated themselves on the patient versions of the CGI-I as responders (4-month mid-test: $73.8 \%$ $(n=59)$ v. 43.4\% $(n=23), z=3.45, P=0.001 ; 8$-month post-test: $75.3 \%(n=64)$ v. $59.6 \%(n=34), \mathrm{z}=1.96, P=0.049)$, however, at the 12-month follow-up the difference was no longer significant (82.6\% $(n=71)$ v. 78.0\% $(n=46), z=0.69, P=0.492)$.

\section{Adequacy of pharmacotherapy}

During the 8 months of the study, 51 (54.3\%) patients in the CSC group and $42(65.6 \%)$ patients in the CAU group used

Table 3 Response and remission rates per treatment programme: intention-to-treat sample

\begin{tabular}{|c|c|c|c|c|c|c|c|c|}
\hline \multirow[b]{3}{*}{ Assessment } & \multicolumn{4}{|c|}{ Anxiety treatment programme, $n(\%)$} & \multicolumn{4}{|c|}{ Depression treatment programme, $n(\%)$} \\
\hline & \multicolumn{2}{|c|}{ Collaborative stepped care group } & \multicolumn{2}{|c|}{ Care as usual group } & \multicolumn{2}{|c|}{ Collaborative stepped care group } & \multicolumn{2}{|c|}{ Care as usual group } \\
\hline & $\begin{array}{l}\text { Response } \\
\quad(n=28)\end{array}$ & $\begin{array}{l}\text { Remission } \\
\quad(n=28)\end{array}$ & $\begin{array}{c}\text { Response } \\
(n=27)\end{array}$ & $\begin{array}{l}\text { Remission } \\
\quad(n=27)\end{array}$ & $\begin{array}{c}\text { Response } \\
(n=46)\end{array}$ & $\begin{array}{l}\text { Remission } \\
\quad(n=45)\end{array}$ & $\begin{array}{c}\text { Response } \\
(n=25)\end{array}$ & $\begin{array}{c}\text { Remission } \\
(n=24)\end{array}$ \\
\hline Mid-test (4 months) & $21(75.0)^{\mathrm{a}}$ & $16(57.1)^{\mathrm{a}}$ & $12(44.4)$ & $7(25.9)$ & $34(73.9)$ & $23(51.1)^{\mathrm{a}}$ & $14(56.0)$ & $6(25.0)$ \\
\hline Post-test (8 months) & $23(82.1)$ & $16(57.1)$ & $18(66.7)$ & $13(48.1)$ & $38(82.6)$ & $26(57.8)$ & $16(64.0)$ & $10(41.7)$ \\
\hline Follow-up (1 year) & $24(85.7)$ & $22(78.6)$ & $22(81.5)$ & $18(66.7)$ & $37(80.4)$ & $30(66.7)$ & $17(68.0)$ & $11(45.8)$ \\
\hline
\end{tabular}


psychopharmaceuticals $(z=-1.36, P=0.173)$. At baseline, 22 (23.4\%) patients in the CSC group and $17(27.4 \%)$ patients in the CAU group were prescribed benzodiazepines $(z=-0.57$, $P=0.571)$. Throughout the study, benzodiazepines were less often prescribed in the CSC group compared with the CAU group (32.3\% ( $n=30)$ v. $51.6 \%(n=33), z=-2.41, P=0.016)$. Also, they were prescribed for a shorter duration in the CSC group compared with the CAU group, resulting in very low benzodiazepine prescription rates at the 8 -month post-test and the 12-month follow-up (6.4\% $(n=6)$ v. 29.0\% $(n=18), z=-3.54, P<0.001)$. Prescription of antidepressants was higher in the CSC group compared with the CAU group $(45.7 \% \quad(n=43)$ v. $36.5 \%$ ( $n=23), z=1.06, P=0.287$ ). The GPs in the CSC group mainly prescribed SSRIs $(81 \%(n=38)$ of the prescriptions), in contrast to the CAU group in which, next to SSRIs $(42 \%(n=10)$ of prescriptions), $46 \%(n=11)$ of the prescriptions consisted of an serotonin noradrenaline reuptake inhibitor or mirtazapine.

\section{Absenteeism from work}

Almost half of the patients reported no sickness absence during the study. About $10 \%$ of the patients reported sickness absence that lasted the entire observation period of 34 weeks, and the remainder $(40 \%)$ reported sickness absence that lasted between 0 and 34 weeks. Patients in the CSC condition reported less sickness absence $(25 \%)$ than patients in the CAU condition (33\%), $z=-1.68, P=0.094)$.

\section{Use of services}

No significant differences between the CSC and CAU condition were found, considering the total number of visits to the primary care physician and mental health workers ( 7.8 v. 7.1, $P=0.490$ ). The intensiveness of care performed by psychiatric nurses in primary care increased significantly in the CSC condition compared with the CAU condition $(3.5$ v. 2.0 visits, $P<0.009)$.

\section{Discussion}

\section{Main findings}

Our results suggest that at the 8-month post-test and the 12 month follow-up CSC and CAU do not differ significantly from each other. However, significant differences in response and remission rates between CSC and CAU after 4 months suggest that the patients treated with CSC experience a faster response and remission of symptoms. In this study, patients in the CAU group also improved considerably, which may reflect a high degree of quality of usual care in our study compared with other collaborative care studies. ${ }^{5}$ Despite the high quality of care, this study shows that the organisation of care in The Netherlands may profit from the rapid provision of low-intensity treatments in primary care and improved collaboration between healthcare providers as established with CSC. Assessment of the patients' perspectives and acceptability showed they generally favoured the CSC model above CAU.

\section{Strengths and limitations}

A strength of this study is that it evaluates a comprehensive stepped-care approach, starting with guided self-help and incorporating primary care and specialised mental healthcare for three highly prevalent classes of mental disorders. The cluster design of the study ensured a naturalistic treatment setting for both CSC and CAU. A limitation of this trial is the smaller number of patients included in the CAU group compared with the CSC group. It was difficult to motivate busy GPs who were randomised to the CAU group to include patients as controls, a problem which has been encountered before in primary care studies. ${ }^{24,25}$ Furthermore, cluster randomisation followed by the selection of patients by GPs carries a risk of selection bias. However, in our study no significant differences at baseline on clinical and demographic variables were found, which may indicate that GPs selected similar patients in each condition.

\section{Comparison with the literature}

The present study is one of the few CSC studies performed to date that includes multiple psychiatric disorders. Compared with most studies describing collaborative care for depression or anxiety, the results from our study are somewhat less promising as we only found a significant difference between CSC and CAU after 4 months. ${ }^{5}$ This is probably as a result of the relatively high response rate in both treatment groups ( $84 \%$ for CSC and $77 \%$ for CAU after 12 months). The high response rate in CAU at follow-up may be a consequence of the natural course of the illnesses, although it is more likely caused by a high level of treatment adequacy by GPs in our care as usual group. ${ }^{2}$ Indeed, a large number $(44 \%)$ of the patients in the CAU group were referred to specialised mental healthcare. A previous study has already demonstrated a similar effectiveness of collaborative care compared with direct referral to specialised mental healthcare. ${ }^{25}$ Furthermore, Patel and colleagues ${ }^{26}$ reported significant effects of collaborative care for common mental disorders in public primary care practices and no effect in private primary care practices, which suggests a diminished effectiveness of collaborative care in systems with a high degree of quality of care.

\section{Anxiety disorder treatment programme}

Disorder-specific secondary outcome measures suggested that treatment effects were most pronounced in the anxiety treatment programme. This is remarkable because more patients in the anxiety treatment programme discontinued treatment compared with patients in the depression treatment programme. The reason for this difference in drop-out rate is unclear. The benefit in the anxiety treatment programme may be explained by the fact that treatment of anxiety disorders in The Netherlands is less adequate than that for depressive disorders. ${ }^{5}$

\section{Use of benzodiazepines}

It is noteworthy that after only a low-intensity intervention consisting of a single educational session and a written prescription manual, the adequacy of benzodiazepine prescribing considerably improved in CSC. Besides the education of GPs, this effect may have resulted from the use of self-help as an alternative to benzodiazepines.

\section{Implications}

This study is the first CSC study including psychotherapeutic interventions and pharmacotherapy in primary and secondary care for common mental disorders. In this study, CSC improved outcomes and quality of care in patients with anxiety, depressive and stress-related disorders, predominantly in the short term. The model justifies replication in larger samples with special attention to its cost-effectiveness. 
Desiree B. Oosterbaan, MD, PhD, Radboud University Nijmegen, Propersona Overwaal Centre for Anxiety Disorders, Nijmegen (at the time of study: Dimence): Marc J. P. M. Verbraak, PhD, Behavioural Science Institute, Department of Clinica Psychology, Radboud University Nijmegen and HSK Group, Arnhem, The Netherlands (at the time of study: Dimence); Berend Terluin, MD, PhD, Department of General Practice and EMGO Institute for Health and Care Research, VU University Medical Center, Amsterdam, The Netherlands; Adriaan W. Hoogendoorn, PhD, Wouter J. Peyrot, MD, Anna Muntingh, PhD, VU University Medical Center and GGZinGeest, Amsterdam, and The Netherlands Institute of Mental Health and Addiction (Trimbos Institute), Utrecht, The Netherlands; Anton J. L. M. van Balkom, MD, PhD, Department of Psychiatry and EMGO Institute for Health and Care Research, vU University Medical Center and GGZinGeest, Amsterdam, The Netherlands

Correspondence: D. B. Oosterbaan, Radboud University, Department of Psychiatry, PO Box 9101, 6500 HB Nijmegen, The Netherlands. Email: d.oosterbaan@psy.umcn.nl

First received 14 Dec 2012, final revision 19 Mar 2013, accepted 27 Mar 2013

\section{Funding}

This study was funded by the Fonds Psychische Gezondheid, The Netherlands.

\section{Acknowledgement}

We thank the patients, primary care providers and mental healthcare workers for their contribution to this study. We owe special thanks to Roelof ten Doesschate, MD, for his inspiration and support.

\section{References}

1 Kovess-Masfety V, Alonso J, Brugha TS, Angermeyer MC, Haro JM, Sevilla-Dedieu C. Differences in lifetime use of services for mental health problems in six European countries. Psychiatr Serv 2007; 58: 213-20.

2 Fernández A, Haro JM, Martinez-Alonso M, Demyttenaere K, Brugha TS, Autonell J, et al. Treatment adequacy for anxiety and depressive disorders in six European countries. Br J Psychiatry 2007; 190: 172-3.

3 Gilbody S, Whitty P, Grimshaw J, Thomas R. Educational and organizational interventions to improve the management of depression in primary care: a systematic review. JAMA 2003; 289: 3145-51.

4 Heideman J, van Rijswijk E, van Lin N, de Loos S, Laurant M, Wensing $M$, et al. Interventions to improve management of anxiety disorders in genera practice: a systematic review. Br J Gen Pract 2005; 55: 867-74.

5 Archer J, Bower P, Gilbody S, Lovell K, Richards D, Gask L, et al. Collaborative care for depression and anxiety problems. Cochrane Database Syst Rev 2012; 10: CD006525.

6 Roy-Byrne P, Craske MG, Sullivan G, Rose RD, Edlund MJ, Lang AJ, et al Delivery of evidence-based treatment for multiple anxiety disorders in primary care: a randomized controlled trial. JAMA 2010; 303: 1921-8.

7 Richards DA, Borglin G. Implementation of psychological therapies for anxiety and depression in routine practice: two year prospective cohort study. J Affect Disord 2011; 133: 51-60.
8 National Institute for Health and Clinical Excellence. Generalised Anxiety Disorder and Panic Disorder (With or Without Agoraphobia) in Adults. Management in Primary, Secondary and Community Care. NICE Clinical Guideline 113. NICE, 2011

9 Woltmann E, Grogan-Kaylor A, Perron B, Georges H, Kilbourne AM, Bauer MS Comparative effectiveness of collaborative chronic care models for menta health conditions across primary, specialty, and behavioral health care settings: systematic review and meta-analysis. Am J Psychiatry 2012; 169 790-804.

10 American Psychiatric Association. Diagnostic and Statistical Manual of Mental Disorders (4th edn) (DSM-IV). APA, 1994.

11 Sheehan DV, Lecrubier $Y$, Sheehan $\mathrm{KH}_{\text {, Amorim }} \mathrm{P}$, Janavs J, Weiller $\mathrm{E}$, et al. The Mini-International Neuropsychiatric Interview (M.I.N.I.): the development and validation of a structured diagnostic psychiatric interview for DSM-IV and ICD-10. J Clin Psychiatry 1998; 59 (suppl 20): 22-33.

12 Verhaak PF, Prins MA, Spreeuwenberg P, Draisma S, van Balkom TJ, Bensing $\mathrm{JM}$, et al. Receiving treatment for common mental disorders. Gen Hosp Psychiatry 2009; 31: 46-55

13 Lewinsohn PM, Antonuccio DO, Steinmetz-Breckenridge JL, Teri L. The Coping with Depression Course: A Psychoeducational Intervention for Unipolar Depression. Castalia Publishing, 1984.

14 White J. 'Stresspac'. A self-help anxiety management package: a controlled outcome study. Behav Cogn Psychother 1995; 23: 89-107.

15 Lewinsohn PM, Clarke GN. Group treatment of depressed individuals: the 'coping with depression' course. Adv Behav Res Ther 1984; 6: 99-114.

16 Guy W. ECDEU Assessment Manual for Psychopharmacology - Revised. NIMH, 1976: 218-22.

17 Hamilton M. The assessment of anxiety states by rating. Br J Med Psychol 1959; 32: $50-5$.

18 Radloff LS. The CES-D scale: a self-report depression scale for research in the general population. Appl Psychol Meas 1977; 1: 401

19 Marks IM, Mathews AM. Brief standard self-rating for phobic patients. Behav Res Ther 1979; 17: 263-7.

20 Derogatis LR, Melisaratos N. The Brief Symptom Inventory: an introductory report. Psychol Med 1983; 13: 595-605.

21 Ware Jr JE, Sherbourne CD. The MOS 36-item short-form health survey (SF 36). I. Conceptual framework and item selection. Med Care 1992; 30: 473-83.

22 Cohen J. Statistical Power Analysis for the Behavioral Science (2nd edn). Lawrence Erlbaum Associates, 1988.

23 Adams G, Gulliford MC, Ukoumunne OC, Eldridge S, Chinn S, Campbell MJ. Patterns of intra-cluster correlation from primary care research to inform study design and analysis. J Clin Epidemiol 2004; 57: 785-94.

24 van Boeijen CA, van Oppen P, van Balkom AJ, visser S, Kempe PT, Blankenstein $\mathrm{N}$, et al. Treatment of anxiety disorders in primary care practice: a randomised controlled trial. Br J Gen Pract 2005; 55: 763-9.

25 van Orden $\mathrm{M}$, Hoffman $\mathrm{T}$, Haffmans J, Spinhoven $\mathrm{P}$, Hoencamp E. Collaborative mental health care versus care as usual in a primary care setting: a randomized controlled trial. Psychiatr Serv 2009; 60: 74-9.

26 Patel V, Weiss HA, Chowdhary N, Naik S, Pednekar S, Chatterjee S, et al. Lay health worker led intervention for depressive and anxiety disorders in India: impact on clinical and disability outcomes over 12 months. Br J Psychiatry 2011; 199: 459-66. 